\title{
Avaliação da qualidade fisiológica de sementes de cultivares de trigo de diferentes partes da espiga
}

Evaluation of the physiological quality of wheat cultivar seeds from different parts of the ear

\section{Evaluación de la calidad fisiológica de semillas de cultivares de} trigo de diferentes partes de la mazorca

Felipe Leandro Felipim Ferrazza ${ }^{1}$

Alex Udich²

Gabriel Toledo Borges ${ }^{3}$

Douglas Tiago Kanieski Jacoboski ${ }^{4}$

Maico Mantovani Tolfo 5

Ricardo Tadeu Paraginski ${ }^{6}$
RECEBIDO EM 21/05/2021

ACEITO EM 10/08/2021

\begin{abstract}
RESUMO
O bom estabelecimento de uma lavoura de trigo e o desempenho inicial das plantas são de extrema importância para elevação dos índices de produtividade da cultura. Nesse sentido, a qualidade fisiológica das sementes é um fator primordial para a implantação de lavouras com maior teto produtivo. Assim, pensando em melhorar a qualidade de sementes disponíveis para utilização no mercado local, o objetivo do trabalho foi avaliar a qualidade de sementes de diferentes cultivares de trigo das partes superior e inferior da espiga. Foram utilizados 12 cultivares de trigo produzidas na safra 2018. Após a maturação fisiológica, foram colhidas e tiveram a espiga dividida em parte superior e inferior, sendo debulhadas e, após, submetidas
\end{abstract}

1 Graduando no Instituto Federal Farroupilha, IFFar, Campus Santo Augusto, Rio Grande do Sul, Brasil. felipe.ferrazza@gmail.com - https://orcid.org/0000-0003-1463-8534

2 Graduando no Instituto Federal Farroupilha, IFFar, Campus Santo Augusto, Rio Grande do Sul, Brasil. alex.udich@gmail.com - https://orcid.org/0000-0002-4325-5893

3 Graduando no Instituto Federal Farroupilha, IFFar, Campus Santo Augusto, Rio Grande do Sul, Brasil. gabriel.toledo@outlook.com - https://orcid.org/0000-0001-8225-5151

4 Graduando no Instituto Federal Farroupilha, IFFar, Campus Santo Augusto, Rio Grande do Sul, Brasil. douglasjacoboski@gmail.com - https://orcid.org/0000-0001-6973-6735

5 Graduando no Instituto Federal Farroupilha, IFFar, Campus Santo Augusto, Rio Grande do Sul, Brasil. maicotolfo07@gmail.com - https://orcid.org/0000-0002-1287-6216 
aos testes de germinação, peso de mil grãos, comprimento de plântulas e massa de plântulas. Os resultados indicam que a parte inferior das espigas apresenta maior peso de mil sementes e maior massa seca de plântulas, já as sementes da parte superior da espiga tiveram um maior percentual de germinação. Existe diferença na qualidade de sementes de diferentes partes da espiga, porém novos trabalhos precisam ser realizados e também métodos de colheita desenvolvidos com essa finalidade.

PALAVRAS-CHAVE: espiga; plântulas; qualidade; sementes.

\section{ABSTRACT}

The good establishment of a wheat crop and the initial performance of the plants are of extreme importance for raising the crop's productivity indices. In this sense, the physiological quality of seeds is a primordial factor for the implantation of crops with a higher productive ceiling. Thus, thinking about improving the quality of seeds available for use in the local market, the objective of this work was to evaluate the quality of seeds of different wheat cultivars from the upper and lower parts of the ear. Twelve wheat cultivars produced in the 2018 crop were used, after physiological maturation they were harvested and had the ear divided into upper and lower parts, threshed and then submitted to germination, thousand grain weight, seedling length and seedling mass tests. The results indicate that the lower part of the ears has a higher weight of a thousand seeds and higher seedling dry mass, whereas the seeds from the upper part of the ear had a higher percentage of germination. There is a difference in the quality of seeds from different parts of the ear, but new work needs to be carried out and also harvesting methods developed for this purpose.

KEYWORDS: spike; seedlings; quality; seeds.

\section{RESUMEN}

El buen asentamiento de un cultivo de trigo y el desempeño inicial de las plantas son de suma importancia para elevar los índices de productividad del cultivo. En este sentido, la calidad fisiológica de las semillas es un factor primordial para la implantación de cultivos con un techo productivo superior. Así, pensando en mejorar la calidad de semillas disponibles para su uso en el mercado local, el objetivo de este trabajo fue evaluar la calidad de semillas de diferentes cultivares de trigo de la parte superior e inferior de la mazorca. Se utilizaron doce cultivares de trigo producidos en la cosecha 2018, tras la la maduración fisiológica se cosecharon y se dividieron las mazorcas en partes superior e inferior, fueron desgranas y luego sometidas a pruebas de germinación, peso de mil granos, longitud de plántula 
y masa de plántula. Los resultados indican que la parte inferior de las mazorcas tiene un mayor peso de mil semillas y mayor masa seca de plántula, mientras que las semillas de la parte superior de la mazorca tienen un mayor porcentaje de germinación. Existe diferencia en la calidad de las semillas de diferentes partes de la mazorca, pero son necesarios nuevos trabajos y el desarrollo de métodos de cosecha para este propósito.

PALABRAS-CLAVE: mazorca; plântulas; calidad; semillas.

\section{Introdução}

A expansão das áreas de produção de trigo no Brasil é de suma importância, tendo em vista que o país é historicamente um dos maiores importadores mundiais do cereal (FAO, 2018). No Brasil, a produção de grãos aumenta a cada ano, principalmente com a elevação do conhecimento dos produtores, que aumentam a produtividade de suas áreas, devido à utilização de novas tecnologias, sementes de alta qualidade, manejo do solo, tratamentos fitossanitários, máquinas e equipamentos agrícolas, aliados à agricultura de precisão (FERRAZZA et al. 2020).

O bom estabelecimento da uma lavoura de trigo e o desempenho inicial das plantas é de extrema importância para elevação dos índices de produtividade da cultura. Segundo Yano et al. (2005), da emergência até a emissão da sétima folha é o período crítico para cultura, sendo as épocas que carecem de uma melhor estrutura, visando enfrentar os diferentes fatores bióticos e abióticos que limitam a sua produtividade.

A qualidade fisiológica da semente é um dos principais atributos que se refletem no aumento da produtividade, influenciam no desempenho inicial da plântula, crescimento e desenvolvimento da cultura (PÁDUA et al., 2010; SZARESKI et al., 2018). Nesse sentido, a qualidade fisiológica das sementes é de extrema importância para a implantação de lavouras com maior crescimento 
inicial de plantas, desempenho de plantas, uniformidade e produtividade de grãos (KOLCHINSKI et al., 2006; CANTARELLI et al., 2015; ABATI et al., 2017). De acordo com Maia (2007), a qualidade fisiológica da semente é determinada no período de sua formação, estando esse processo e o acúmulo de reservas estreitamente correlacionados com a intensidade fotossintética da planta e com o fluxo de assimilados para os órgãos reprodutivos.

Trabalhos realizados em nível de lavoura indicam que o uso de sementes de alto vigor apresenta, também, um potencial maior de produção, chegando a índices de até $10 \%$ de aumento de produtividade (FRANÇA-NETO et al., 2012). De acordo com Carvalho et al. (2015), poucos estudos têm mostrado a influência do tamanho da semente no aspecto físico e fisiológico de atributos do trigo, bem como a relação canônica entre esses caracteres de interesse agronômico. Ainda, trabalhos mostram que ocorre grande variação dos atributos fisiológicos entre cultivares de trigo e lotes da mesma cultivar (PRANDO et al., 2012), e sementes classificadas em tamanhos diferentes apresentam variabilidade nos atributos fisiológicos (KEHL et al., 2016; DEMARI et al., 2016). Trabalho realizado por Demari et al. (2021) afirma que existe variabilidade dos atributos físicos e fisiológicos devido às dimensões das sementes de trigo, sendo estes específicos para os efeitos de lotes de cultivares e sementes, porém o mesmo não avaliou a qualidade de sementes de diferentes partes da espiga. Assim, considerando a qualidade de sementes como parte dos componentes que garantem o rendimento da cultura do trigo e o cenário atual da produção de trigo na região na busca por mais informações sobre possíveis melhorias na qualidade de sementes, o objetivo do trabalho foi avaliar a qualidade de sementes de trigo da parte inferior e superior da espiga, visando à obtenção de sementes de elevada qualidade fisiológica. 


\section{Material e métodos}

A pesquisa é um estudo realizado com o objetivo de encontrar respostas aos problemas que são propostos, sendo desenvolvida com inúmeras fases, desde a formulação adequada do problema até a apresentação adequada de resultados. O trabalho foi realizado no Laboratório de Fitotecnia do Instituto Federal de Educação, Ciência e Tecnologia Farroupilha - Campus Santo Augusto, onde foram utilizados 12 cultivares de trigo colhidos após a maturação fisiológica nos municípios de Santo Augusto e Nova Ramada, Rio Grande do Sul, provenientes de lavouras comerciais. As cultivares utilizadas foram TBIO Ponteiro (Lote 1), TBIO Sossego (Lote 2), BRS Reponte (Lote 3), TBIO Aton (Lote 4), TBIO Alpaca (Lote 5), TBIO Energix 201 (Lote 6), TBIO Astro (Lote 7), TBIO Toruk (Lote 8), TBIO Audaz (Lote 9), Coodetec CD 1303 (Lote 10), TBIO Sonic (Lote 11) e TBIO Energia I (Lote 12). Após a colheita, as sementes foram secas em estufa a $35^{\circ} \mathrm{C}$, no Laboratório, até obtenção da umidade de $13 \%$ para padronização e separadas manualmente em parte superior e inferior. Posteriormente, foi realizada a debulha manual para serem submetidas às avaliações:

I Peso de mil sementes (PMS): Determinado com contagem de oito repetições de 100 sementes e pesagem em balança analítica (Brasil, 2009), e os resultados expressos em gramas;

I Teste de germinação: A avaliação do teor de germinação foi conduzida em quatro repetições de 100 sementes, em substrato de papel, em germinador regulado a $20^{\circ} \mathrm{C}$, embebido em água na quantidade de 2,5 vezes o peso do substrato seco, visando ao adequado umedecimento. As contagens foram realizadas ao $4^{\circ}$ dia (primeira contagem) e ao $8^{\circ}$ dia (contagem final) após a semeadura, seguindo as Regras para Análise de Sementes (BRASIL, 2009). Os resultados foram expressos em percentagem, obtida pela média das repetições; 
I Comprimento de plântulas: A avaliação do comprimento de plântula foi realizada ao final do teste de germinação, quando foi efetuada a medida das partes das plântulas emergidas, partes aéreas e radiculares, em plântulas normais, com auxílio de uma régua, e os resultados foram expressos em centímetros;

I Massa de plântulas: A massa verde foi realizada através da pesagem das plântulas após o teste de germinação, e a massa seca das plântulas foi realizada através da secagem das plântulas normais obtidas a partir dos testes de germinação (contagem final). As repetições de cada amostra foram acondicionadas em sacos de papel, identificados e levados à estufa com circulação de ar forçada, mantida à temperatura de $60^{\circ} \mathrm{C}$, por um período de 24 horas (NAKAGAWA, 1999). Após esse período, cada repetição teve a massa avaliada em balança com precisão de 0,0001g, e os resultados médios foram expressos em miligramas por plântula;

I Análise estatística: Os resultados foram submetidos à análise de variância ANOVA, e os efeitos do tratamento de sementes foram avaliados pelo teste de Tukey $(p<0,05)$, com o programa SASM - Agri (2001).

\section{Resultados e discussões}

Os resultados do peso de mil sementes (Tabela 1 ) indicaram que a parte inferior da espiga de todas as cultivares apresentou valores mais elevados quando comparada à parte superior. Dentre as cultivares, a TBIO Sonic foi a que demostrou o maior PMS, tanto na parte superior, quanto inferior, 37,69 gramas e 43,87 gramas, respectivamente, destacando-se nesse parâmetro produtivo. A média de todas as cultivares da parte superior foi de 30,50 gramas e da parte inferior 34,29 gramas. Os resultados sugerem que a diferença de 3,79 gramas (11,05\%) superior para as sementes da base da espiga deve-se 
à maior translocação de seiva para os grãos inferiores e, muitas vezes, as enzimas sintetizadoras de carboidratos, principais constituintes dos grãos, aproveitam todos os metabólitos para a parte inferior da espiga, reduzindo a disponibilidade para a parte superior.

A amilose é sintetizada por ADPglucose pirofosforilase (AGPase) e amido ligado a grânulos sintase (GBSS), e a amilopectina é sintetizada pelo coordenado de ações da AGPase, amido sintase solúvel (SS), enzima ramificadora do amido enzima (BE) e enzima de desramificação do amido (DBE), conforme descrito em trabalhos de Dian et al. (2005) e Hwang et al. (2005), e acredita-se que esse processo pode ser mais intenso na parte basal da espiga de trigo. Ainda, de acordo com Buratto et al. (2020), a folha bandeira é considerada a fonte primária para o enchimento de grãos, devido à curta distância da espiga, sendo as folhas situadas na porção superior do colmo responsáveis pela maior parte da produção de fotoassimilados, que realizam a produção de grãos. E a possível menor translocação na espiga pode ter resultado nesses dados. 
TABELA 1 - Peso de mil grãos (gramas) da parte superior e inferior da espiga de diferentes cultivares de trigo.

\begin{tabular}{|c|c|c|c|c|c|c|c|c|}
\hline \multirow{2}{*}{ Cultivar $^{a}$} & \multicolumn{7}{|c|}{ Parte da espiga } & \multirow{2}{*}{ C.v. } \\
\hline & \multicolumn{3}{|c|}{ Superior } & \multicolumn{4}{|c|}{ Inferior } & \\
\hline TBIO Ponteiro & $33,99 \pm$ & $0,08 b$ & B & 35,86 & \pm & $0,04 \mathrm{~b}$ & $A$ & $2,41 \%$ \\
\hline TBIO Sossego & $31,78 \pm$ & $0,09 \mathrm{~cd}$ & B & 34,37 & \pm & 0,09 bc & A & $3,37 \%$ \\
\hline BRS Reponte & $27,70 \pm$ & $0,05 \mathrm{fg}$ & B & 29,55 & \pm & $0,09 \mathrm{e}$ & A & $3,10 \%$ \\
\hline TBIO Aton & $32,10 \pm$ & $0,19 \mathrm{bcd}$ & $A$ & 35,13 & \pm & $0,24 b$ & $A$ & $8,83 \%$ \\
\hline TBIO Alpaca & $27,51 \pm$ & $0,09 \mathrm{fg}$ & B & 31,61 & \pm & 0,07 de & A & $3,62 \%$ \\
\hline TBIO Energix 201 & $30,35 \pm$ & 0,06 de & B & 33,66 & \pm & $0,05 \mathrm{bcd}$ & A & $2,27 \%$ \\
\hline TBIO Astro & $33,15 \pm$ & $0,07 \mathrm{bcd}$ & B & 42,27 & \pm & 0,19 a & A & $5,41 \%$ \\
\hline TBIO Audaz & $28,40 \pm$ & 0,06 efg & B & 33,53 & \pm & 0,07 bcd & A & $3,20 \%$ \\
\hline Coodetec CD 1303 & $28,22 \pm$ & $0,04 \mathrm{fg}$ & B & 31,69 & \pm & 0,20 cde & A & $7,75 \%$ \\
\hline TBIO Sonic & $37,69 \pm$ & 0,10 a & B & 43,87 & \pm & 0,07 a & A & $2,41 \%$ \\
\hline TBIO Energia I & $29,27 \pm$ & 0,06 ef & B & 30,95 & \pm & 0,03 de & A & $2,18 \%$ \\
\hline TBIO Toruk & $26,93 \pm$ & $0,08 \mathrm{~g}$ & B & 29,01 & \pm & 0,06 e & A & 26,93 \\
\hline Média & & 30,59 & & & & 34,29 & & \\
\hline C.V. & & $3,98 \%$ & & & & $4,74 \%$ & & \\
\hline
\end{tabular}

Nota: ' Médias aritméticas \pm o Desvio Padrão seguidos por letras maiúsculas iguais na mesma linha e minúsculas iguais na mesma coluna, não diferem entre si pelo teste de Tukey $(p \leq 0,05)$.

Fonte: Próprios Autores.

A germinação da semente é a característica fisiológica que indica o potencial de reproduzir uma nova planta e é condicionada pelo desenvolvimento regular do embrião e pela quantidade de reservas disponíveis, sendo esses pré-requisitos necessários para o desenvolvimento de uma plântula vigorosa e uma planta bem desenvolvida (ADEBISI et al., 2013). Os resultados da Tabela 2 indicam que houve variação na porcentagem de germinação aos quatro dias entre as cultivares, seja na parte superior ou inferior da espiga. A avaliação entre parte superior e inferior, avaliado individualmente para cada cultivar, em sua maior parte não apresentou diferença estatística para 7 cultivares aos 4 dias. Em trabalho realizado por Demari et al. (2021), o índice de velocidade de germinação para um cultivar mostrou que as sementes da peneira> 3,0 mm (lotes C, D, E e F), peneira 2,0 a 2,49 mm (lote A e B) e peneira $<2,0 \mathrm{~mm}$ (lotes A, B e C) apresentaram uma redução desse caráter. 
Os resultados do teste de germinação final, com a contagem final (Tabela 2), indicam que a germinação foi maior na parte superior da espiga na maioria das cultivares, e a parte superior da espiga apresentou menor peso de mil sementes (Tabela 1), indicando que não existe relação de maior peso com maior percentual de germinação de sementes. De acordo com Moreira et al. (2016), sementes menores necessitam de uma menor quantidade de água para iniciar o processo germinativo, favorecendo a rápida digestão de reservas e translocação para o eixo embrionário, estando de acordo com os resultados obtidos neste trabalho. A utilização de sementes de alto desempenho fisiológico possibilita maior velocidade de germinação e emergência, além de melhor estabelecimento das plantas a campo (LIMA et al., 2006).

De acordo com Peripolli et al. (2019), sementes de soja maiores têm menor porcentual de germinação. Em sementes de sansão-do-campo (Mimosa caesalpiniifolia), os maiores tamanhos de semente proporcionaram maior massa seca de raiz (ALVES et al., 2005). Sementes menores de açafrão-bastardo (Carthamus tinctorius) apresentaram maior índice de velocidade de germinação, em relação àquelas de tamanho maior (SADEGHI et al., 2011). De acordo com Demari et al. (2021), ao avaliar diferentes tamanhos de sementes, identificou que em geral a resposta ao tamanho da semente de trigo variou de acordo com as cultivares, sendo superior em vigor e germinação, primeira contagem de germinação, índice de velocidade de germinação e emergência a campo, comprimento da raiz da plântula e massa seca da parte aérea.

Para Marcos Filho (2015), o comportamento fisiológico das sementes está relacionado ao genótipo, entretanto, Costa et al. (2005) relataram que o ambiente de cultivo pode influenciar essa característica, conforme proporciona melhores ou piores condições climáticas às plantas durante a maturação das sementes. Ressalta-se que condições ambientais e de genótipos podem afetar 
a translocação de fotoassimilidos nas diferentes partes da espiga, estando de acordo com os resultados obtidos.

TABELA 2 - Porcentagem de germinação aos quatro dias e aos oito dias de sementes da parte superior e inferior da espiga de diferentes cultivares de trigo.

\begin{tabular}{|c|c|c|c|c|}
\hline \multirow{3}{*}{ Cultivara } & \multicolumn{2}{|c|}{ Germinação aos 4 dias } & \multicolumn{2}{|c|}{ Germinação aos 8 dias } \\
\hline & \multicolumn{2}{|c|}{ Parte da espiga } & \multicolumn{2}{|c|}{ Parte da espiga } \\
\hline & Superior & Inferior & Superior & Inferior \\
\hline TBIO Ponteiro & $32,00 \mathrm{~cd} A$ & 17,00 de $B$ & 76,50 abc $A$ & 62,00 bcd $B$ \\
\hline TBIO Sossego & 6,00 f $B$ & 14,50 de $A$ & $57,00 \mathrm{~cd} \quad \mathrm{~A}$ & 52,50 d $A$ \\
\hline BRS Reponte & 72,00 ab A & 51,50 ab $B$ & 75,50 abc $A$ & $66,50 \mathrm{bcd} A$ \\
\hline TBIO Aton & $41,50 \mathrm{~cd} A$ & 40,00 bc $A$ & $78,50 \quad a b \quad A$ & 64,00 bcd $B$ \\
\hline TBIO Alpaca & $33,00 \mathrm{~cd} A$ & 37,50 bc $A$ & 76,00 abc A & 74,50 bc $A$ \\
\hline TBIO Energix 201 & 20,50 def $A$ & $29,50 \mathrm{~cd} \quad \mathrm{~A}$ & 85,00 a $A$ & 79,50 ab $A$ \\
\hline TBIO Astro & 25,50 def $B$ & 46,00 bc $A$ & 85,50 a $B$ & 97,00 a $A$ \\
\hline TBIO Audaz & 18,50 def $A$ & 15,50 de $A$ & 59,00 bcd A & $61,00 \mathrm{bcd} A$ \\
\hline Coodetec CD 1303 & 83,00 ab $A$ & 65,50 a $A$ & 86,00 a $A$ & 65,50 bcd $B$ \\
\hline TBIO Sonic & $10,00 \quad f \quad A$ & 6,50 e $A$ & 72,00 abcd $A$ & $65,50 \mathrm{bcd} A$ \\
\hline TBIO Energia I & $8,50 \quad f \quad A$ & 4,50 e $A$ & 60,50 bcd A & $48,50 \quad d \quad B$ \\
\hline TBIO Toruk & $60,50 \quad b \quad A$ & 41,50 bc $B$ & $64,50 \mathrm{bcd} A$ & $50,50 \quad d \quad B$ \\
\hline Média & 34,25 & 30,79 & 73 & 65,58 \\
\hline C.V. & $19,11 \%$ & $24,76 \%$ & $10,96 \%$ & $11,87 \%$ \\
\hline
\end{tabular}

Nota: ${ }^{a}$ Médias aritméticas seguidas por letras maiúsculas iguais na mesma linha e minúsculas iguais na mesma coluna, não diferem entre si pelo teste de Tukey $(p \leq 0,05)$.

Fonte: Próprios Autores.

Os resultados de comprimento de plântulas da parte aérea (Tabela 3) indicam que não houve diferença entre parte superior e inferior, com exceção das cultivares TBIO Aton e TBIO Toruk, em que as sementes da parte inferior da espiga originaram plântulas com parte aérea maior. Entre as cultivares, a cultivar BRS Reponte foi a que apresentou maior comprimento de parte aérea de plântulas com as sementes extraídas da parte superior da espiga, e da parte inferior, a cultivar TBIO Toruk foi a que mais se destacou. O comprimento da raiz das plântulas (Tabela 3) indica que não houve diferença entre as partes superior e inferior da espiga, com exceção das cultivares TBIO Alpaca e TBIO 
Toruk, em que a parte inferior foi maior e, na cultivar Coodetec CD 1303, a parte superior da espiga teve um maior comprimento radicular de plântulas.

TABELA 3 - Comprimento da parte aérea $(\mathrm{cm})$ e parte radicular $(\mathrm{cm})$ de plântulas da parte superior e inferior da espiga de diferentes cultivares de trigo.

\begin{tabular}{|c|c|c|c|c|}
\hline \multirow{3}{*}{ Cultivar } & \multicolumn{2}{|c|}{$\begin{array}{l}\text { Comprimento da parte } \\
\text { aérea de plântulas }\end{array}$} & \multicolumn{2}{|c|}{$\begin{array}{l}\text { Comprimento da parte } \\
\text { radicular de plântulas }\end{array}$} \\
\hline & \multicolumn{2}{|c|}{ Parte da espiga } & \multicolumn{2}{|c|}{ Parte da espiga } \\
\hline & Superior & Inferior & Superior & Inferior \\
\hline TBIO Ponteiro & 5,83 cde $A$ & 4,80 efg $A$ & 11,35 abc $A$ & $13,03 \mathrm{ab} \quad \mathrm{A}$ \\
\hline TBIO Sossego & 4,25 de $A$ & 5,03 defg A & $9,56 \quad C \quad A$ & 11,20 bc $A$ \\
\hline BRS Reponte & 10,18 a $A$ & 9,70 ab $A$ & 13,23 ab A & $12,93 \quad a b \quad A$ \\
\hline TBIO Aton & 5,58 cde B & 7,38 abcde $A$ & 11,95 abc $A$ & $13,33 \mathrm{ab} \quad \mathrm{A}$ \\
\hline TBIO Alpaca & 7,33 bc $A$ & 9,01 abc $A$ & 12,83 abc B & 15,60 a $A$ \\
\hline TBIO Energix 201 & 5,40 cde $A$ & 6,70 cdefg A & 11,58 abc $A$ & 12,15 abc $A$ \\
\hline TBIO Astro & 5,38 cde A & 4,85 efg $A$ & 10,00 bc $A$ & 11,10 bc $A$ \\
\hline TBIO Audaz & 5,69 cde $A$ & 5,15 defg $A$ & 11,43 abc $A$ & 10,96 bc $A$ \\
\hline Coodetec CD 1303 & 8,51 ab $A$ & 7,80 abcd $A$ & 11,73 abc $A$ & $8,95 \quad C \quad B$ \\
\hline TBIO Sonic & 3,51 e $A$ & $3,93 \quad g \quad A$ & 10,39 abc A & 10,23 bc $A$ \\
\hline TBIO Energia I & 4,25 de $A$ & 4,54 efg $A$ & 11,25 abc $A$ & $12,55 \mathrm{abc} A$ \\
\hline TBIO Toruk & 7,63 bc $B$ & 10,13 a $A$ & $9,45 \quad C \quad B$ & 11,35 bc $A$ \\
\hline Média & 6,13 & 6,59 & 11,23 & 11,95 \\
\hline C.V. & $38,09 \%$ & $41,95 \%$ & $28,12 \%$ & $30,28 \%$ \\
\hline
\end{tabular}

Nota: ${ }^{a}$ Médias aritméticas seguidas por letras maiúsculas iguais na mesma linha e minúsculas iguais na mesma coluna, não diferem entre si pelo teste de Tukey $(p \leq 0,05)$.

\section{Fonte: Próprios Autores.}

Alguns trabalhos realizados afirmam que sementes mais vigorosas proporcionam emergência de plântulas mais rápida e uniforme, favorecem o crescimento da parte aérea e das raízes, e reduzem o índice de plantas subdesenvolvidas, conferindo maior tolerância a adversidades ambientais e elevam o potencial produtivo (FINCH-SAVAGE; BASSEL, 2015; MARCOS FILHO, 2015; KRZYZANOWSKI et al., 2018). A mensuração do comprimento, bem como a determinação da matéria seca das plântulas proporcionam estimativas do vigor das sementes, visto que sementes mais vigorosas possuem maior capacidade de nutrir o eixo embrionário, resultando em plântulas com maiores taxas de 
crescimento e de acumulação de matéria seca (DAN et al., 1987). Conforme descrito por Rigoli et al. (2009), sementes de trigo maiores tendem a aumentar o comprimento de plântula e indiretamente expressar alto vigor, e plantas com maior desenvolvimento inicial apresentam qualidade fisiológica superior, bem como capacidade competitiva.

Os resultados de massa verde de plântulas (Tabela 4) indicaram que houve diferença entre as cultivares TBIO Aton, TBIO Energix 201 e TBIO Sonic, em que a parte inferior da espiga mostrou resultados maiores de massa verde. Já quanto à diferença entre cultivares, na parte superior, a cultivar TBIO Alpaca teve o maior índice e, na parte inferior, as cultivares TBIO Aton, TBIO Alpaca e TBIO Astro foram superiores às demais. Os valores de massa seca de plântulas (Tabela 4) indicam que a parte inferior das espigas gerou plântulas com maior massa seca, com exceções nas cultivares TBIO Sossego, TBIO Aton, TBIO Alpaca, TBIO Audaz, Coodetec CD 1303, TBIO Energia I e TBIO Toruk, que não apresentaram diferença estatística. Já a cultivar TBIO Sonic foi a que apresentou maior massa seca na parte superior e inferior entre as cultivares.

A massa seca é um parâmetro eficiente para avaliarvigor, pois quanto maior o acúmulo de matéria seca, mais vigorosa é a plântula (BISOGNIN et al., 2016). Dentre as características físicas das sementes que afetam o desempenho das plântulas, o tamanho é um dos componentes a influenciar o estabelecimento da cultura (AMBIKA et al., 2014), sendo seu efeito atribuído ao fato de sementes maiores possuírem elevada quantidade de reservas, o que pode explicar o aumento no crescimento e no vigor de plântulas (FINCH-SAVAGE; BASSEL, 2016). Os resultados estão de acordo com o peso de mil sementes, sendo que a parte inferior da espiga contém as sementes maiores e, conforme Pereira et al. (2008), para a cultura do tamarindo (Tamarindus indica), sementes grandes e muito grandes produzem plantas de maior diâmetro de coleto, maior 
massa seca de parte aérea e raiz. Portanto, a classificação das sementes é uma técnica que deve ser recomendada.

TABELA 4 - Massa verde (gramas) e massa seca (gramas) de plântulas da parte superior e inferior da espiga de diferentes cultivares de trigo.

\begin{tabular}{|c|c|c|c|c|}
\hline \multirow{3}{*}{ Cultivar ${ }^{a}$} & \multicolumn{2}{|c|}{ Massa verde de plântulas (gramas) } & \multicolumn{2}{|c|}{ Massa seca de plântulas (gramas) } \\
\hline & \multicolumn{2}{|c|}{ Parte da espiga } & \multicolumn{2}{|c|}{ Parte da espiga } \\
\hline & Superior & Inferior & Superior & Inferior \\
\hline TBIO Ponteiro & 0,58 bcd $A$ & $0,64 \operatorname{abcd} A$ & 0,11 bcd $B$ & 0,15 abc $A$ \\
\hline TBIO Sossego & $0,51 \mathrm{~cd} \quad \mathrm{~A}$ & 0,56 bcd $A$ & 0,13 abc $A$ & 0,13 bcd $A$ \\
\hline BRS Reponte & $0,59 \mathrm{bcd} A$ & 0,59 bcd $A$ & $0,10 \mathrm{~cd} \quad \mathrm{~B}$ & 0,11 de $A$ \\
\hline TBIO Aton & 0,61 bc $B$ & 0,82 a $A$ & 0,12 bcd $A$ & 0,13 bcd $A$ \\
\hline TBIO Alpaca & 0,80 a $A$ & 0,80 a $A$ & 0,11 bcd $A$ & $0,12 \mathrm{~cd} \quad \mathrm{~A}$ \\
\hline TBIO Energix 201 & 0,55 bcd B & 0,74 ab $A$ & 0,11 bcd $B$ & 0,13 bcd $A$ \\
\hline TBIO Astro & $0,70 \quad a b \quad A$ & 0,79 a $A$ & 0,13 ab $B$ & 0,16 ab $A$ \\
\hline TBIO Audaz & 0,59 bcd $A$ & $0,53 \mathrm{~cd} \quad \mathrm{~A}$ & $0,12 \operatorname{bcd} A$ & $0,14 \mathrm{bcd} A$ \\
\hline Coodetec CD 1303 & $0,56 \mathrm{bcd} A$ & $0,52 \mathrm{~cd} \quad \mathrm{~A}$ & $0,10 \mathrm{~cd} \quad \mathrm{~A}$ & 0,11 de $A$ \\
\hline TBIO Sonic & 0,61 bcd $B$ & 0,69 abc A & 0,15 a $B$ & 0,17 a $A$ \\
\hline TBIO Energia I & $0,49 \mathrm{~cd} \quad \mathrm{~A}$ & $0,48 \quad d \quad A$ & 0,13 abc A & $0,13 \mathrm{~cd} \quad \mathrm{~A}$ \\
\hline TBIO Toruk & $0,47 \quad \mathrm{~cd} \quad \mathrm{~A}$ & $0,51 \mathrm{~cd} \quad \mathrm{~A}$ & $0,09 \quad d \quad A$ & 0,08 e $A$ \\
\hline Média & 0,59 & 0,64 & 0,12 & 0,13 \\
\hline C.V. & $10,90 \%$ & $12,37 \%$ & $10,74 \%$ & $10,26 \%$ \\
\hline \multicolumn{5}{|c|}{ Nota: ${ }^{a}$ Médias aritméticas seguidas por letras maiúsculas iguais na mesma linha e minúsculas iguais na } \\
\hline
\end{tabular}

Fonte: Próprios Autores.

\section{Conclusões}

Os resultados indicam que a parte inferior das espigas de trigo apresentam maior peso de mil sementes e maior massa seca de plântulas, já as sementes da parte superior da espiga tiveram uma maior porcentagem de germinação, na primeira e segunda contagem, entretanto os valores foram baixos. Novos trabalhos precisam ser realizados avaliando a qualidade de sementes de diferentes partes da espiga e diâmetro, produzidas nas mesmas condições edafoclimáticas, a fim de gerar informações para futuros processos de produção de sementes de alta qualidade. 


\section{Agradecimentos}

Gostaríamos de agradecer ao CNPq (Conselho Nacional de Desenvolvimento Científico e Tecnológico), à FAPERGS (Fundação de Amparo à Pesquisa do Estado do Rio Grande do Sul) e ao Instituto Federal de Educação, Ciência e Tecnologia Farroupilha - Campus Santo Augusto. 


\section{Referências}

ABATI, J.; BRZEZINSKI, C.R.; FOLONI, J.S.S.; ZUCARELI, C.; BASSOI, M.C.; HENNING, F.A. Seedling emergence and yield performance of wheat cultivars depending on seed vigor and sowing density. Journal of Seed Science, v. 39, n. 1, p. 66-74, 2017.

ADEBISI, M.A.; KEHINDE, T.O.; SALAU, A.W.; OKESOLA, L.A.; PORBENI, J.B.O.; ESURUOSO, A.O.; OYEKALE, K.O. Influence of different seed size fractions on seed germination, seedling emergence and seed yield characters in tropical soybean (Glycine max L. Merrill). International Journal of Agricultural Research, v.8, n.1, p.26-33, 2013.

ALVES, E.U.; BRUNO, R.L.A.; OLIVEIRA, A.P.; ALVES, A.U.; PAULA, R.C. Influência do tamanho e da procedência de sementes de Mimosa caesalpiniifolia Benth sobre a germinação e vigor. Revista Árvore, v. 29, n.6, p.877-885, 2005.

AMBIKA, S.; MANONMANI, V.; SOMASUNDAR, G. Review on effect of seed size on seedling vigour and seed yield. Research Journal of Seed Science, v.7, n.2, p.31-38, 2014.

BISOGNIN, M.B.; KULCZYNSKI, S.M.; FERRARI, M.; GAVIRAGHI, R.; PELEGRIN, A.J.; SOUZA, V.Q. Desempenho fisiológico de sementes olerícolas em diferentes tempos de hidrocondicionamento. Revista de Ciências Agrárias, v. 39, n. 3, p. 349-359, 2016.

BRASIL. Ministério da Agricultura, Pecuária e Abastecimento. Regras para análise de sementes. Ministério da Agricultura, Pecuária e Abastecimento. Secretaria de Defesa Agropecuária. Brasília, DF: Mapa/ACS, 2009. 398p.

BURATTO, Juliana Sawada; ROSA, Jean Carlos Gomes; DE FREITAS VANZO, Aline Taiane. Correlação entre tamanho de espiga e outros caracteres morfológicos em triticale. Brazilian Journal of Animal and Environmental Research, v. 3, n. 3, p. 2775-2781, 2020.

CANTARELLI, L.D.; SCHUCH, L.O.B.; TAVARES, L.C.; RUFINO, C.A. Variabilidade de plantas de soja originadas de sementes de diferentes níveis de qualidade fisiológica. Acta Agronómica, v. 64, n. 3, p. 234-238, 2015

CANTERI, M. G., ALTHAUS, R. A., VIRGENS FILHO, J. S., GIGLIOTI, E. A., GODOY, C. V. SASM - Agri: Sistema para análise e separação de médias em experimentos agrícolas pelos métodos Scoft - Knott, Tukey e Duncan. Revista Brasileira de Agrocomputação, v.1, n.2, p.18-24. 2001.

CARVALHO, I.R.; SOUZA, V.Q.; NARDINO, D.N.; SCHMIDT, D.; BARETTA, D. Correlações canônicas entre caracteres morfológicos e componentes de produção em trigo de duplo propósito. Pesquisa Agropecuária Brasileira, v.50, n.8, 2015.

COSTA, N.P.; MESQUITA, C.M.; MAURINA, A.C.; FRANÇA NETO, J.B.; KRZYZANOWSKI, F.C.; OLIVEIRA, M.C.N.; HENNING, A.A. Perfil dos aspectos físicos, fisiológicos e químicos de sementes de soja produzidas em seis regiões do Brasil. Revista Brasileira de Sementes, Jaboticabal, v.27, n.2, p.01-06, 2005.

DAN, E. L. et al. Transferência de matéria seca como método de avaliação do vigor de sementes de soja. Revista Brasileira de Sementes, v. 9, n. 3, p. 45-55, 1987. 
DEMARI, G.H.; CARVALHO, I.R.; SZARESKI, V.J.; DELLAGOSTIN, S.M.; LORO, M.V.; SOUZA, V.Q.; ZIMMER, P.D.; VILLELA, F.A.; PEDÓ, T.; AUMONDE, T.Z.; Seed classification: Influence on the vigor expression in Triticum aestivum L.. Revista Brasileira de Agropecuária Sustentável (RBAS), v. 11, n. 1, p. 43-56, Março, 2021.

DEMARI, GH.; CARVALHO, I.R.; NARDINO, M.; SZARESKI, V.J.; DATSCH, A.C.; PELEGRIN, A.J.; MARTINS, T.; SANTOS, N.L.; LAUTENCHLEGER, F.; PEDO, T.; SOUZA, V.Q.; AUMONDE, T.Z.; BASSO, C.J.; ZIMMER, P.D. Poultry litter as an alternative source for nitrogen in wheat. International Journal of Current Research, v.8, p.39733-39737, 2016.

DIAN, W. H. JIANG, P. WU, Evolution and expression analysis of starch synthase III and IV in rice. Journal of Experimental Botany, 56, 623e632, 2005.

FAO. 2018. Food and Agriculture Organization of the United Nations. Food Outlook: biannual report on global food markets. Roma: FAO. 169p.

FERRAZZA, F. L. F..; JACOBOSKI, D. T. K. .; WYREPKOWSKI, A.; RODRIGUES, L.; FIGUEIRÓ, A. G.; PARAGINSKI, R. T. Seed quality and productive parameters of soybean seeds submitted to different seed treatments before sowing. Research, Society and Development, [S. I.], v. 9, n. 9, p. e47996232, 2020. DOI: 10.33448/rsd-v9i9.6232. Disponível em: https://www.rsdjournal.org/index. php/rsd/article/view/6232. Acesso em: 24 sep. 2021.

FINCH-SAVAGE, W. E.; BASSEL, G. W. Seed vigour and crop establishment: extend-ing performance beyond adaptation. Journal of Experimental Botany, v. 67, p. 1-25, 2015.

FINCH-SAVAGE, W. E.; BASSEL, G. W. Seed vigour and crop establishment: Extending performance beyond adaptation. Journal of Experimental Botany, v.67, n.3, p.567-591, 2016.

FRANÇA-NETO, J.B.; KRZYZANOWSKI, F.C.; HENNING, A.A. Plantas de alto desempenho e a produtividade da soja. Seed News, Pelotas, Pelotas, v.16, n.6, p.8-11, nov./dez. 2012.

HWANG, J.W.; KIM, S.K.; LEE, J.S.; KIM, I.S. Gene expression of the biosynthetic enzymes and biosynthesis of starch during rice grain development. Journal of Plant Biology, 48, 448e455, 2005.

KEHL, K.; KEHL, K.; SZARESKI, V.J.; CARVALHO, I.R.; NARDINO, M.; DEMARI, GH.; ROSA, T.C.; GUTKOSKI, L.C.; PEDO, T.; AUMONDE, T.Z.; SOUZA, V.Q.; ZIMMER, P.D.; MENEGHELLO, G.E. Genotype environment interaction under industrial and physiological quality of wheat seeds. International Journal of Current Research, v.8, p.38461-38468, 2016.

KOLCHINSKI, E.M.; SCHUCH, L.O.B.; PESKE, S.T. Crescimento inicial de soja em função do vigor de sementes. Revista Brasileira de Agrociência, v. 12, n. 2, p. 163-166, 2006.

KRZYZANOWSKI, F. C.; FRANÇA-NETO, J. B.; HENNING, A. A. A alta qualidade da semente de soja: fator importante para a produção da cultura. Londrina: Embrapa Soja, 2018. 24 p. (Embrapa Soja. Circular técnica, 136).

LIMA, T. C.; MEDINA, P. F.; FANAN, S. Avaliação do vigor de sementes de trigo pelo teste de enveIhecimento acelerado. Revista Brasileira de Sementes, v. 28, p. 106-113, 2006. 
MAIA, A.R. Envelhecimento acelerado e avaliação da qualidade fisiológica de sementes de trigo acondicionadas em diferentes embalagens e armazenadas em ambiente natural em Ibitirama-ES. Dissertação de Mestrado. Programa de Pós-Graduação em Produção Vegetal da Universidade Federal do Espírito Santo, 2007.

MARCOS FILHO, J. Fisiologia de sementes de plantas cultivadas. 2. ed. Londrina: Abrates, 2015. $660 \mathrm{p}$.

MOREIRA, F. J. C.; DA SILVA, M. A. P. \& MEDEIROS FILHO, S. Germinação e crescimento inicial de cajuí (Anacardium microcarpum Ducke) em função do tamanho das sementes e do tempo de embebição. Cadernos de Cultura e Ciência, v. 15, n. 1, p. 19-28, 2016. Disponível em: <http://dx.doi. org/10.1590/S0100-6762200600060000>.

NAKAGAWA, J. Testes de vigor baseados no desempenho das plântulas. In: Krzyzanoski, F.C.; Vieira, R.D.; França Neto, J.B. (Ed.). Vigor de sementes: conceitos e testes. Londrina: ABRATES, 1999. p. 2.1-2.24

PÁDUA, G.P.; ZITO, R.K.; ARANTES, N.E.; NETO, J.D.B.F. Influência do tamanho da semente na qualidade fisiológica e na produtividade da cultura da soja. Revista Brasileira de Sementes, v.32, n.3, p.9-16, 2010.

PEREIRA, P. C.; FREITAS, R. S.; MELO, B.; ALMEIDA, A.; PEREIRA, A. P.; SANTANA, G.; MAGNO, J.; LUZ, Q. Influência do tamanho de sementes na qualidade de mudas de tamarindeiro. Bioscience Journal, v.24, n.4, p.73-79, 2008.

PERIPOLLI, M., MARTINI SANCHOTENE, D., DOS SANTOS LIMA, C., POZZATO CRISTOFARI, L., PIVETTA, M., MASSUQUINI CONCEIÇÃO, G., \& FÁVERO ROSADO, G. (2019). Qualidade fisiológica de sementes de soja provenientes de dois tamanhos de peneira. Vivências, 15(29), 267-278. Disponível em: https://doi.org/10.31512/vivencias.v15i29.70

PRANDO, A.M.; ZUCARELI, C.; FRONZA, V.; OLIVEIRA, E.A.P.; PANOFF, B. Formas de ureia e doses de nitrogênio em cobertura na qualidade fisiológica de sementes de trigo. Revista Brasileira de Sementes, v.34, n.2, 2012.

RIGOLI, R.P.; AGOSTINETTO, D.; VAZ DA SILVA, J.M.B.; FONTABA, L.C.; VARGAS, L. Potencial competitivo de cultivares de trigo em função do tempo de emergência. Planta Daninha, Viçosa-MG, v.27, n.1, p.41-47, 2019.

SADEGHI, H.; KHAZAEI, F.; SHEIDAEI, S.; YARI, L. Effect of seed size on seed germination behavior of safflower (Carthamus tinctorius L.). Journal of Agricultural and Biological Science, v.6, n.4. p.5-8, 2011.

SZARESKI, V.J.; CARVALHO, I.R.; KEHL, K.; LEVIEN, A.M.; ROSA, T.C.; BARBOSA, M.H.; DEMARI, G.H.; PIMENTEL, J.R.; TROYJACK, C.; MARTINAZZO, E.G.; SOUZA, V.Q.; VILLELA, F.A.; PEDO, T.; AUMONDE, T. Z. Wheat seeds yield in Brazil: Phenotypic and predicted genetic approaches for genotype ranking. Genetics and Molecular Research, v.17, p.1-13, 2018.

YANO, G.T.; TAKAHASHI, H.W.; WATANABE, T.S. Avaliação de fontes de nitrogênio e épocas de aplicação em cobertura para o cultivo do trigo. Semina: Ciências Agrárias, v. 26, n. 2, p. 141-148, 2005. 